\title{
Endoscopic submucosal dissection for gastric tube carcinoma after esophagectomy contributes to long-term outcomes: a retrospective case series
}

Satoru Hashimoto ( $\nabla$ hashim@med.niigata-u.ac.jp )

Niigata Nbiversity Medical and Dental Hospital https://orcid.org/0000-0002-1418-6382

Hiroki Sato

Niigata University

Ken-ichi Mizuno

Niigata University

Kazuya Takahashi

Niigata University

Masafumi Takatsuna

Niigata University

Junji Yokoyama

Niigata University

Hiroshi Ichikawa

Niigata University

Manabu Takeuchi

Nagaoka Red Cross Hospital

Masaaki Kobayashi

Niigata Cancer Center Hospital

Shuji Terai

Niigata University

Research article

Keywords: gastric tube carcinoma, gastric cancer, endoscopic submucosal dissection, esophagectomy

Posted Date: October 21st, 2020

DOl: https://doi.org/10.21203/rs.3.rs-50700/v2

License: (1) (1) This work is licensed under a Creative Commons Attribution 4.0 International License.

Read Full License 


\section{Abstract}

Background: Gastric tube carcinoma (GTC) after esophagectomy has been observed increasingly in recent years after advanced treatment of esophageal carcinoma. Surgical removal of the reconstructed gastric tube is not usually preferred; however, endoscopic submucosal dissection (ESD) is greatly anticipated to be a promising option. This study investigated the efficacy and safety of ESD for GTC.

Methods: This study was designed based on a retrospective survey performed at our single center between 2003 and 2018. Patients with GTC were treated with ESD and followed-up according to the policy of our institution. The curability of ESD was evaluated histologically according to the Japanese gastric cancer treatment guidelines. Patient characteristics, outcomes of ESD procedures, and long-term outcomes were analyzed.

Results: A total of 31 patients ( 2 females; median age, 73 years) with 45 GTC cases were treated with ESD. The mean period from esophagectomy to the diagnosis of GTC was 10.6 years. The only adverse event due to ESD was bleeding (2 patients: $6.5 \%$ ). No fatal events, such as perforation, occurred. The rates of complete resections and curative resections were $80.6 \%$ and $48.4 \%$, respectively. The 3-year and 5 -year overall survival rates were $67.6 \%$ and $47.7 \%$, respectively. The 3-year and 5-year disease-specific survival rates were $100 \%$ and $92.9 \%$, respectively. One patient died of GTC and 14 patients died of other diseases, including five primary carcinomas.

Conclusions: ESD for GTC can be a safe treatment and contribute to positive long-term outcomes. Longterm regular gastroscopy is required for early detection. Moreover, additional care should be given to GTC patients who are at high risk for other primary carcinomas or comorbidities.

\section{Background}

Esophagectomy is a curative treatment for esophageal squamous cell carcinoma; to repair the removed section of the esophagus, a reconstructed gastric tube can be used [1]. However, post-esophagectomy patients are at high risk for gastric tube carcinoma (GTC). Recently, it has been observed that the progress of treatments for esophageal carcinoma has also led to an increase in the occurrence of GTC after esophagectomy. Based on data published by Sugiura and colleagues, GTC prevalence seems to have increased over the years [2]. Additionally, a high incidence rate of gastric carcinoma due to elevated numbers of Helicobacter pylori infections, particularly in Asian countries, including Japan, remains a major medical issue [3] [4].

Surgical removal of the reconstructed gastric tube has been reported as a severe burden for patients with GTC because of its associated high mortality [2]. Endoscopic submucosal dissection (ESD) was developed as a minimally invasive treatment for early gastrointestinal carcinoma [5], and it has been attempted for GTC as well [6] [7] [8]. However, the technical difficulty of the procedure increases greatly with fibrotic changes of the suture line due to surgical reconstruction [9] (Fig. 1), limited working space, and worsened maneuverability of the endoscope. Reports focused on ESD for GTC are limited. Hence, we 
attempted to identify the clinical outcomes of ESD for GTC by analyzing our clinical experience with these cases.

\section{Methods}

\section{Patients}

All patients who underwent ESD for GTC after esophagectomy between 2003 and 2018 at the Niigata University Medical and Dental Hospital were recruited. ESD indications for GTC in our institution were based on the 2010 Japanese gastric cancer treatment guidelines[10]. The absolute indication was a differentiated-type intramucosal cancer $\leq 2 \mathrm{~cm}$ in size without ulceration, and the expanded indications were as follow: 1) differentiated-type intramucosal cancer $>2 \mathrm{~cm}$ in size without ulceration, 2) differentiated-type intramucosal cancer $\leq 3 \mathrm{~cm}$ with ulceration, and 3) undifferentiated-type intramucosal cancer $\leq 2 \mathrm{~cm}$ in size without ulceration. However, in cases where it was possible to perform complete resection, ESD was performed for patients who had a risk of surgery without indication. Before performing complete resection, endoscopic ultrasonography (EUS) was done for some patients suspected of having an SM2 invasion. If the EUS revealed no rupture in the submucosa layer, ESD was considered as a possible treatment.

Atrophic gastritis in the gastric tube was diagnosed endoscopically, according to the Kimura-Takemoto classification [11]. This study was designed based on a retrospective survey performed at our single center and was conducted as part of a clinical study approved by our Institutional Review Board. This study was also performed in accordance with the Declaration of Helsinki.

\section{ESD procedure}

ESD was performed by several experts with more than 6 years of experience with the procedure. Patients were sedated by an intravenous injection of propofol or midazolam; their blood pressure, electrocardiography results, oxygen saturation, and bispectral index were monitored during the ESD procedure. All ESD procedures were performed with an upper gastrointestinal endoscope (GIF-Q260J; Olympus Medical Systems, Tokyo, Japan), a standard electrosurgical generator (VIO300D or ICC200; ERBE, Tübingen, Germany), and a Hook knife (KD-260LR; Olympus Medical Systems). Carbon dioxide insufflation was used during the procedure. Mucosal marking around the tumor margin was performed circumferentially with the Hook knife. Glycerol (10\% glycerin and $5 \%$ fructose) was injected into the submucosa (SM) to elevate the lesion. Bleeding vessels were coagulated using monopolar Coagrasper Hemostatic Forceps (ED-410LR; Olympus Medical Systems). When the tumor was localized on the suture line, the submucosal dissection was performed, starting from the peripheral area and continuing to the suture line connecting the fibrotic submucosal layer, while paying careful attention to avoiding damage to the muscle layer because the clear submucosal layer is lost in the area of the suture line (Fig. 2). Fig. 3 shows a representative case of GTC on the suture line. For severe fibrosis on the suture line, attaching an ST hood short-type (DH-28GR; Fujifilm, Tokyo, Japan) or clipping using the line method was performed to create effective countertraction and obtain good visualization [12]. 
Histopathological assessment

The resected specimens were fastened on boards using pins and fixed in $10 \%$ formalin. Serial-step sections at 2-mm intervals were made after 24 hours, stained with hematoxylin and eosin, and evaluated by pathologists. The tumor size, depth of invasion, lymphovascular invasion, presence of ulceration, horizontal margin involvement, and vertical margin involvement were evaluated.

At our institution, the curability of ESD for GTC was evaluated based on the 2010 Japanese gastric cancer treatment guidelines [10]. Complete resection was defined as when en bloc resection with tumorfree horizontal and vertical margins was achieved. Next, the curability of the specimens removed with complete resection was evaluated. Curative resection was defined as when the complete resection specimens were without lymphovascular invasion and fit into one of the following categories: a) histologically differentiated-type pT1a and ulceration-negative; b) histologically differentiated-type pT1a, ulceration-positive, and tumor size $\leq 3 \mathrm{~cm} ; \mathrm{c}$ ) histologically undifferentiated-type $\mathrm{pT} 1 \mathrm{a}$, ulcerationnegative, and tumor size $\leq 2 \mathrm{~cm}$; and d) histologically differentiated-type pT1b (SM1, <500 $\mu \mathrm{m}$ from the muscularis mucosa) and tumor size $\leq 3 \mathrm{~cm}$. A tumor comprising components of both differentiated-type and undifferentiated-type carcinomas was classified according to the quantitative predominance. Noncurative resection was defined as when the specimen could not be diagnosed as a curative resection.

\section{Follow-up after ESD}

For patients who had a curative resection, an upper endoscopy was performed annually at regular intervals. However, for patients who had a non-curative resection, an additional repeat ESD or surgical resection was discussed. If additional treatment was difficult because of the tumor's high invasiveness, upper endoscopy and computed tomography were performed every 6 months.

\section{Statistical analysis}

Patient characteristics, histological results of ESD specimens, and clinical outcomes, including procedurerelated outcomes, clinical course, and long-term outcomes, were analyzed. Regarding patients' demographics and histological findings, continuous variables are expressed as median and range; noncontinuous data are expressed as a percentage. Kaplan-Meier analyses were used to estimate the cumulative survival of patients. Statistical analyses were performed using Statistical Package for Social Science (SPSS) version 24 (IBM SPSS Inc., Chicago, IL, USA).

\section{Results}

\section{Patient characteristics and ESD procedure-related outcomes}

Patient characteristics and procedure-related outcomes are shown in Table 1. A total of 31 consecutive patients (29 men and 2 women) with 45 GTC cases were treated using ESD. The median age of the patients was 73 years (range, 58-84 years) at the time of ESD. Six patients had multiple lesions with synchronous occurrence, and nine patients had multiple lesions with metachronous occurrence. The 
median period from esophagectomy to ESD after the initial GTC was 10.6 years (range, 0.8-18.2 years). The route of reconstruction was retrosternal for 16 cases and the posterior mediastinum for 15 cases. In our series, all patients with GTC had Helicobacter pylori-related atrophic gastritis.

Table 1: Patient characteristics and ESD procedure-related outcomes $(n=31)$

\begin{tabular}{lc}
\hline \multicolumn{2}{c}{ Patient characteristics } \\
\hline Sex ratio (\%) & Male \\
Female & $29(93.5)$ \\
Median age, years (range) & $2(6.5)$ \\
Synchronous/metachronous occurrence & $73(58-84)$ \\
Period from onset to esophagectomy, years (range) & $6 / 9$ \\
Reconstruction route & $10.6(0.8-18.2)$ \\
Retrosternal & 16 \\
Posterior mediastinum & 15 \\
Atrophic gastritis $\quad$ Positive & 31 \\
Negative & 0 \\
ESD procedure-related outcomes & \\
Adverse events (\%) Bleeding & $2(6.5)$ \\
Perforation & 0 \\
Complete resection (\%) & $25(80.6)$ \\
Curative resection (\%) & $15(48.4)$ \\
\hline
\end{tabular}

ESD, endoscopic submucosa dissection.

The only adverse event due to ESD was bleeding (2 patients: 6.5\%). Fatal complications, including perforation, were not observed. The rates of complete resection and curative resection were $80.6 \%$, and $48.4 \%$, respectively.

\section{Clinical and histopathological findings}

Histopathological findings are shown in Table 2. In terms of tumor location, there were 26 lesions in the lower gastric tube, 17 lesions in the middle gastric tube, and 2 lesions in the upper gastric tube. Four lesions were on the suture line. Macroscopically, 34 lesions were diagnosed as 0-llc (slightly depressed type), 10 lesions were diagnosed as 0-lla (slightly elevated type), and one lesion was diagnosed as 0-I (elevated type). The median tumor size was $17.5 \mathrm{~mm}$ (range, 5-53 mm), with 33 differentiated, 2 undifferentiated, and 10 mixed-type adenocarcinomas. The depth of tumor invasion was pT1a (mucosa [M]) in 29 lesions, pT1b (SM1: $<500 \mu \mathrm{m}$ below the muscularis mucosa in the SM) in 3 lesions, pT1b (SM2: $500 \mu \mathrm{m}$ or deeper invasion from the SM) in 12 lesions, and pT2 (muscularis propria [MP]) in one lesion. Lymphovascular invasion was observed with seven lesions. The horizontal margin was positive in one lesion and the vertical margin was positive in five lesions. The positive horizontal margin was misdiagnosed when there was a poorly differentiated lesion. The positive vertical margin was diagnosed because of its invasion deeper than SM2.

Table 2: Clinical and histological findings of gastric tube carcinomas (n₫45) 


\begin{tabular}{|c|c|c|}
\hline \multirow{2}{*}{\multicolumn{3}{|c|}{ Location }} \\
\hline & Upner & 2 \\
\hline & Middle & 17 \\
\hline & Lower & 26 \\
\hline On the s & line & 4 \\
\hline \multirow{3}{*}{ Macroscopic types } & & \\
\hline & 0 -IIa & 10 \\
\hline & 0 -IIC & \\
\hline \multicolumn{2}{|c|}{ Median size of the tumors, $\mathrm{mm}$} & $17.5(5-53)$ \\
\hline \multirow{3}{*}{ Histological type } & Differentiated (tub1 tub2) & 33 \\
\hline & Undifferentiated (por, sig) & 2 \\
\hline & Mixed & 10 \\
\hline \multirow{4}{*}{ Depth of invasion } & pT1a (M) & 29 \\
\hline & pT1b (SM1) & 3 \\
\hline & pT1b (SM2) & 12 \\
\hline \multirow{2}{*}{\multicolumn{3}{|c|}{ Lymphovascular invasion }} \\
\hline & Positive & 7 \\
\hline \multirow{3}{*}{ Horizontal margin } & Negative & 38 \\
\hline & Positive & 1 \\
\hline & Negative & 44 \\
\hline \multirow{2}{*}{ Vertical margin } & Positive & 5 \\
\hline & Negative & 40 \\
\hline
\end{tabular}

tub1, well-differentiated adenocarcinoma; tub2, moderately-differentiated adenocarcinoma; por, poorly-differentiated adenocarcinoma; sig, signet-ring cell carcinoma; M, mucosal; SM1, $<500 \mu \mathrm{m}$ below the muscularis mucosa into the submucosa; SM2, $500 \mu \mathrm{m}$ or deeper invasion from the muscularis mucosa into the submucosa.

\section{Clinical course after ESD and long-term outcomes}

All patients were followed-up for a median of 50 months (range, 2-168 months). Twenty-nine patients were followed-up during this study $(93.5 \% ; 29 / 31)$. The clinical course details are shown in Fig. 4. Fourteen patients (48.3\%) had curative resection and 15 patients $(51.7 \%)$ had non-curative resection. Among the 14 patients with curative resection, 8 patients were alive, and 6 patients died of another disease (pneumonia, 3; hypopharyngeal cancer, 1 ; cervical esophageal cancer, 1 ; sepsis due to psoas abscess, 1$)$. Metachronous occurrence was diagnosed in three patients $(21.2 \% ; 3 / 14)$ and curative ESD was performed for all patients. No patients with curative resection died of GTC.

Of the 15 patients with non-curative resection, one had an undifferentiated mucosal lesion, five had positive vertical margins, one had only a positive horizontal margin, and eight had negative tumor margins with lymphovascular invasion or invasion more than SM2. All were followed-up without additional surgery. The patient with an undifferentiated mucosal lesion larger than $20 \mathrm{~mm}$ was alive. Of the five patients with positive vertical margins, four patients had SM2 invasion, and one had MP invasion. Two patients with SM2 invasion were alive, including one patient who had local recurrence and 
underwent additional radiation. The other two patients with SM2 invasion died of intrahepatic cholangiocarcinoma and heart failure, respectively. The patient with MP invasion died of local recurrence, despite chemotherapy (5-fluorouracil and cisplatin) after ESD. The one patient with only a positive horizontal margin died of pancreatic cancer. Three patients among the eight patients who had negative tumor margins with lymphovascular invasion or invasion more than SM2 were alive, including one patient who was treated with curative ESD for metachronous lesions after non-curative ESD. The other five patients died of another disease (pneumonia, 2; hypopharyngeal cancer, 1; malignant lymphoma, 1 ; heart failure, 1).

The 3-year and 5-year overall survival rates were $67.6 \%$ and $47.7 \%$, respectively. The 3-year and 5-year disease-specific survival rates were $100 \%$ and $92.9 \%$, respectively (Fig. 5). Only one patient died of GTC resected with non-curative ESD. Of the 14 deaths due to other causes, 5 were other primary carcinomas (hypopharyngeal cancer, 2; cervical esophageal cancer, 1; pancreatic cancer, 1; malignant lymphoma, 1).

\section{Discussion}

Surgical resection of the reconstructed gastric tube for GTC is invasive and is associated with high mortality [2] [13]. ESD, which is a minimally invasive treatment for early gastric cancer, has an important role in the treatment of GTC. Some studies have previously reported the long-term outcomes of ESD for GTC [6] [8] [14] [15]. However, all of them were designed based on a retrospective survey at a single center. Although a multicenter prospective study is desirable, it is important to collect clinical data from each institution because GTC is not frequently encountered. Similarly, this study is a retrospective survey at a single center; however, our clinical data included a comparative number of cases (31 patients and 45 lesions) and a relatively long follow-up period (median, 50 months). As there are very few patients with GTC, even in highly specialized hospitals, retrospective data from each institution is valuable, and collecting this data makes it possible to understand the efficacy and safety of ESD for GTC. Another advantage of our study is that we report the long-term outcomes of patients with non-curative resection (15 patients).

Some lesions that were not indicated for ESD according to the Japanese gastric cancer guidelines [10] were included in this study because of their surgical complications. This study found that the rate of complete ESD resections for GTC after esophagectomy was $80.6 \%$. Lesions with incomplete resection included one lesion with a positive lateral margin and five lesions with positive vertical margins. The reason for the positive lateral margin was misdiagnosis of the tumor margin because of undifferentiated adenocarcinoma. The size of this lesion was larger than $40 \mathrm{~mm}$; therefore, ESD was not indicated. We considered that several negative biopsy samples from outside the lesion should have been confirmed before ESD. This patient died of pancreatic cancer 30 months after ESD without GTC recurrence.

All five lesions with positive vertical margins had deep invasion to the SM or MP. The patient with invasion to the MP had local recurrence and died despite chemotherapy. One patient with invasion to the $\mathrm{SM}$ also had local recurrence and received additional radiation. Because the follow-up term after 
radiation was short in this case, remnant recurrence is possible. There have been no reports on the efficacy of chemotherapy or radiation for local recurrence of GTC after ESD. Although total reconstruction gastrectomy has been considered the standard treatment for local massive recurrence after ESD, surgery is not usually preferred because of the high risk of complications, such as leakage and pneumonia [2]. Thoracoscopic and endoscopic cooperative surgery has been reported to be minimally invasive [16], and the development of other surgical methods with less invasive techniques is expected for advanced GTC.

Only one patient died of GTC during this investigation. The disease-specific survival rate was more than $92.9 \%$, which included patients with non-curative resection. The result was considered a permissible level. However, 14 deaths due to other causes were confirmed, and the 5-year overall survival rate was $47.7 \%$. Because patients were elderly (mean age, 73 years) and already had esophageal and gastric cancer, they were considered at high risk for other primary carcinomas. Moreover, they may have developed respiratory and cardiovascular diseases due to smoking, diabetes mellitus, or hyperlipidemia. ESD is less invasive than surgery for patients with GTC. We considered that ESD for GTC might be tolerable for patients with SM2 invasion in the event that complete resection is feasible. However, it should not be performed for patients with MP invasion. EUS may be performed to confirm the possibility of complete resection if necessary.

Although ESD for GTC is safe and effective [6] [8], it is more difficult to resect lesions than early gastric cancer without reconstruction. The gastric tube has limited working space and can cause food retention and fluid pooling due to a notable deformity of the stomach and severe fibrosis on the suture line after surgical reconstruction [9]. Therefore, ESD for GTC should be performed by fully trained endoscopists. When there is a lesion on the suture line, severe fibrosis of the submucosal layer, and remaining surgical staples, attaching the ST hood short-type and traction methods, such as clipping with the line method, are effective for obtaining good endoscopic visualization [12] [17] [18]. When a lesion is submerged in fluid or blood, we should consider positioning the patient in the opposite direction. We consider ESD for GTC a safe treatment because adverse events (delayed bleeding) occurred in only two cases. However, careful attention to distinctive complications, such as precordial skin burns after ESD in cases involving the retrosternal route, is required [19].

All patients had Helicobacter pylori-related atrophic gastritis. Because they were at high risk for gastric cancer, it is important to detect GTC during the early stage [20]. Furthermore, we must stay aware of metachronous occurrences after curative ESD. Recent remarkable advances in magnifying endoscopy and narrow band imaging systems have allowed the development of early treatments for gastric carcinomas [21]. Careful observation is needed to manage worsening conditions in the gastric tube, such as deformity of the stomach, bleeding, and fluid pooling. The period from esophagectomy to GTC was 10.6 years in this study; therefore, regular long-term follow up is necessary.

This study had several limitations. First, our observations are based on an analysis of retrospective data from a single center; therefore, treatments were not based on any clear protocols. Second, the analysis 
was performed over a long period; therefore, the skill of the endoscopist who performed the diagnosis and ESD procedure for GTC gradually improved, which might have affected the results.

\section{Conclusions}

In conclusion, ESD for GTC can be a safe treatment and contribute to good long-term outcomes. Longterm regular gastroscopy is required for early detection. Moreover, additional care should be given to GTC patients who are at high risk for other primary carcinomas or comorbidities.

\section{Abbreviations}

GTC: gastric tube carcinoma; ESD: endoscopic submucosal dissection; EUS: endoscopic ultrasonography; M: mucosa; SM: submucosa; MP: muscularis propria

\section{Declarations}

\section{Ethics approval and consent to participate}

The study was approved by the Institutional Review Board at Niigata University.

\section{Consent for publication}

Not applicable.

\section{Availability of data and materials}

The datasets used and analyzed during the current study are available from the corresponding author on reasonable request.

\section{Competing interests}

The authors declare that they have no competing interests.

\section{Funding}

There are no funding sources to declare.

\section{Author's contributions}

$\mathrm{SH}, \mathrm{HS}, \mathrm{KM}, \mathrm{KT}$, and MT collected data from patient charts and contributed to manuscript writing and analyses. SH, HS, KM, KT, MT, and MK contributed to performing ESD. JY, HI, and ST supervised the study and contributed to revising the manuscript. The authors read and approved the final manuscript. 
Not applicable.

\section{References}

1. Zhang W, Yu D, Peng J, Xu J, Wei Y. Gastric-tube versus whole-stomach esophagectomy for esophageal cancer: A systematic review and meta-analysis. PLoS One. 2017, 12(3):e0173416.

2. Sugiura $T$, Kato $H$, Tachimori $Y$, Igaki $H$, Yamaguchi $H$, Nakanishi $Y$. Second primary carcinoma in the gastric tube constructed as an esophageal substitute after esophagectomy. J Am Coll Surg. 2002;194(5):578-83.

3. Uemura N, Okamoto S, Yamamoto S, Matsumura N, Yamaguchi S, Yamakido M, Taniyama K, Sasaki $\mathrm{N}$, Schlemper RJ. Helicobacter pylori infection and the development of gastric cancer. N Engl J Med. 2001;345(11):784-9.

4. Fukase K, Kato M, Kikuchi S, Inoue K, Uemura N, Okamoto S, Terao S, Amagai K, Hayashi S, Asaka M. Effect of eradication of Helicobacter pylori on incidence of metachronous gastric carcinoma after endoscopic resection of early gastric cancer: an open-label, randomised controlled trial. Lancet (London, England). 2008;372(9636):392-7.

5. Ono H, Yao K, Fujishiro M, Oda I, Nimura S, Yahagi N, et al. Guidelines for endoscopic submucosal dissection and endoscopic mucosal resection for early gastric cancer. Dig Endosc. 2016;28(1):3-15.

6. Nonaka S, Oda I, Sato C, Abe S, Suzuki H, Yoshinaga S, et al. Endoscopic submucosal dissection for gastric tube cancer after esophagectomy. Gastrointest Endosc. 2014;79(2):260-70.

7. Mukasa M, Takedatsu H, Matsuo K, Sumie H, Yoshida H, Hinosaka A, et al. Clinical characteristics and management of gastric tube cancer with endoscopic submucosal dissection. World $\mathrm{J}$ Gastroenterol. 2015;21(3):919-25.

8. Watanabe K, Hikichi T, Nakamura J, Hashimoto M, Takagi T, Suzuki R, et al. Clinical outcomes and adverse events of endoscopic submucosal dissection for gastric tube cancer after esophagectomy. Gastroenterol Res Pract. 2019;2019:2836860.

9. Tawaraya S, Jin M, Matsuhashi T, Suzuki Y, Sawaguchi M, Watanabe N, et al. Advanced feasibility of endoscopic submucosal dissection for the treatment of gastric tube cancer after esophagectomy. Gastrointest Endosc. 2014;79(3):525-30.

10. Japanese gastric cancer treatment guidelines 2010 (ver. 3). Gastric cancer. 2011;14(2):113-23.

11. Kimura K, Satoh K, Ido K, Taniguchi Y, Takimoto T, Takemoto T. Gastritis in the Japanese stomach. Scand J Gastroenterol Suppl. 1996;214:17-20; discussion 21-13.

12. Oyama T. Counter traction makes endoscopic submucosal dissection easier. Clin Endosc. 2012;45(4):375-8.

13. Bamba T, Kosugi S, Takeuchi M, Kobayashi M, Kanda T, Matsuki A, et al. Surveillance and treatment for second primary cancer in the gastric tube after radical esophagectomy. Surg Endosc 2010;24(6):1310-7. 
14. Shirakawa Y, Noma K, Maeda N, Ninomiya T, Tanabe S, Kikuchi S, et al. Clinical characteristics and management of gastric tube cancer after esophagectomy. Esophagus. 2018;15(3):180-9.

15. Hirayama Y, Fujisaki J, Yoshimizu S, Horiuchi Y, Yoshio T, Ishiyama A, et al. Efficacy and safety of endoscopic resection for gastric tube cancer after surgical resection of esophageal squamous cell carcinoma. Esophagus. 2019;16(2):194-200.

16. Tsujii Y, Yamasaki M, Hayashi Y, Tanaka K, Makino T, Doki Y, et al. Thoracoscopic and endoscopic cooperative surgery (TECS): a novel less invasive technique for resection of gastric tube cancer after esophagectomy. Endoscopy. 2019;51(1):E5-e6.

17. Yamamoto $\mathrm{H}$. Endoscopic submucosal dissection of early cancers and large flat adenomas. Clin Gastroenterol Hepatol. 2005;3(7 Suppl 1):S74-6.

18. Oguri N, Ikeya T, Shiratori Y. Endoscopic submucosal dissection using a traction method for gastric tube cancer. Dig Endosc. 2020;32(3):e52-e53.

19. Miyagi M, Yoshio T, Hirasawa T, Ishiyama A, Yamamoto $Y$, Tsuchida T, et al. Precordial skin burns after endoscopic submucosal dissection for gastric tube cancer. Dig Endosc. 2015;27(7):742-6.

20. Okamoto N, Ozawa S, Kitagawa Y, Shimizu Y, Kitajima M. Metachronous gastric carcinoma from a gastric tube after radical surgery for esophageal carcinoma. Ann Thorac Surg. 2004;77(4):1189-92.

21. Hayee B, Inoue H, Sato H, Santi EG, Yoshida A, Onimaru M, et al. Magnification narrow-band imaging for the diagnosis of early gastric cancer: a review of the Japanese literature for the Western endoscopist. Gastrointest Endosc. 2013;78(3):452-61

\section{Figures}




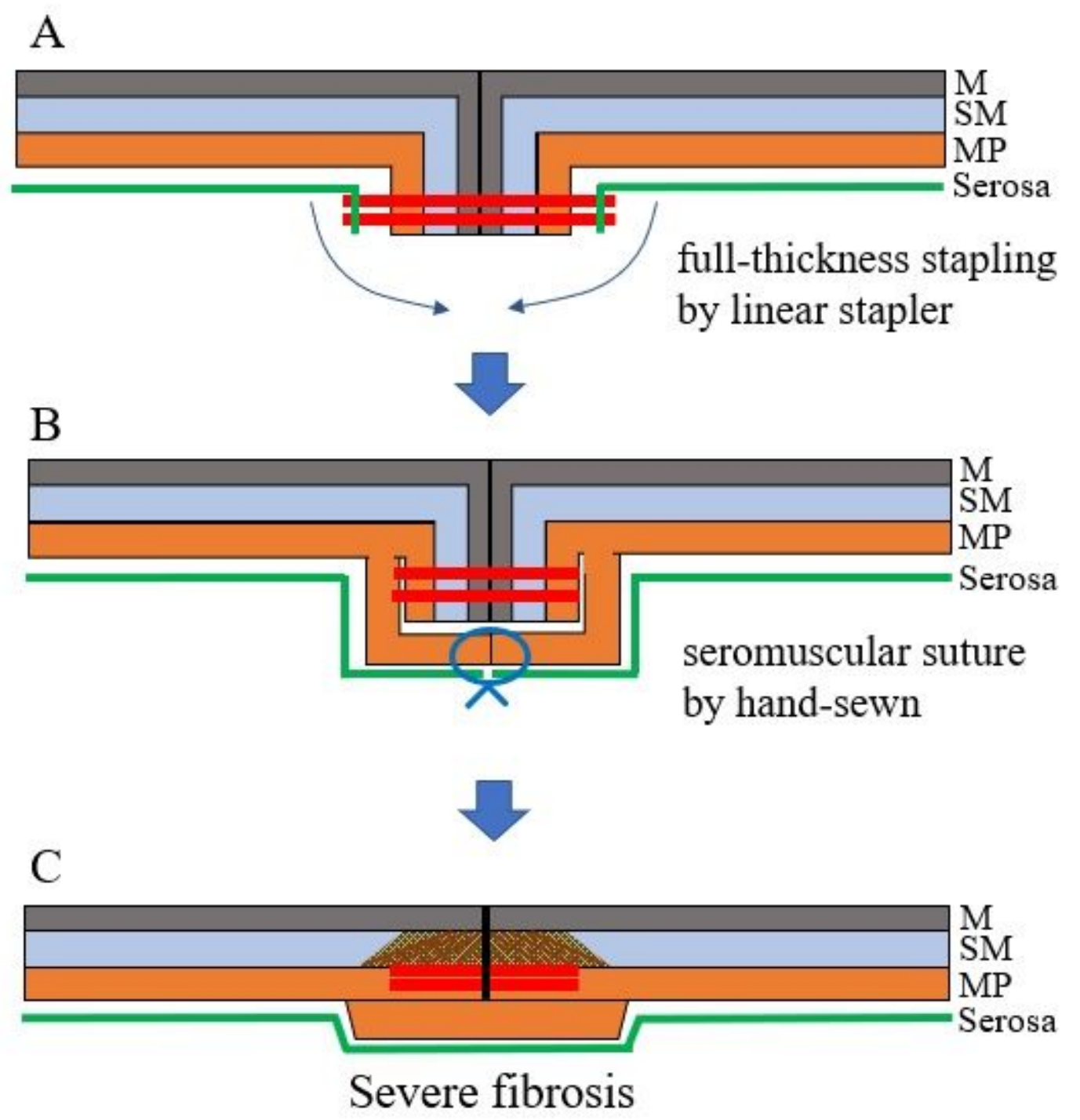

M: mucosa; SM: submucosa; MP: muscularis mucosa

Figure 1

Sectional view of gastric tube reconstruction (full-thickness suture). (A) First, full-thickness suturing is performed with a linear stapler. (B) Second, seromuscular suturing is performed by hand to reinforce the suture. (C) Submucosa around the suture line with severe fibrosis. 

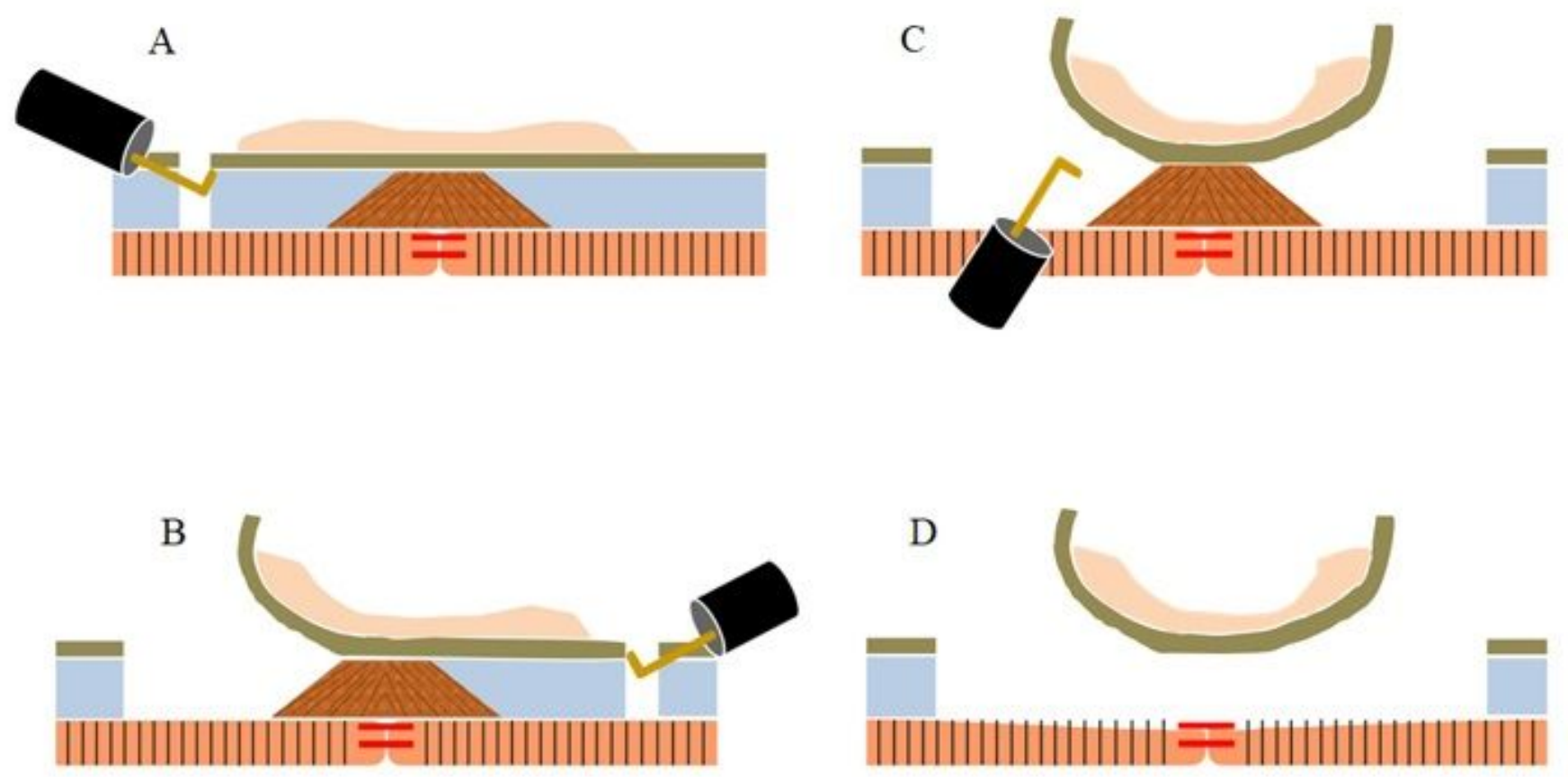

Figure 2

Strategy for gastric tube carcinoma on the suture line. (A and B) Mucosal incision and submucosal dissection starting from the peripheral and continuing to the suture line. ( $C$ and $D)$ Using good countertraction, the connecting fibrotic submucosal layer is dissected while focusing careful attention on not damaging the muscle layer. 


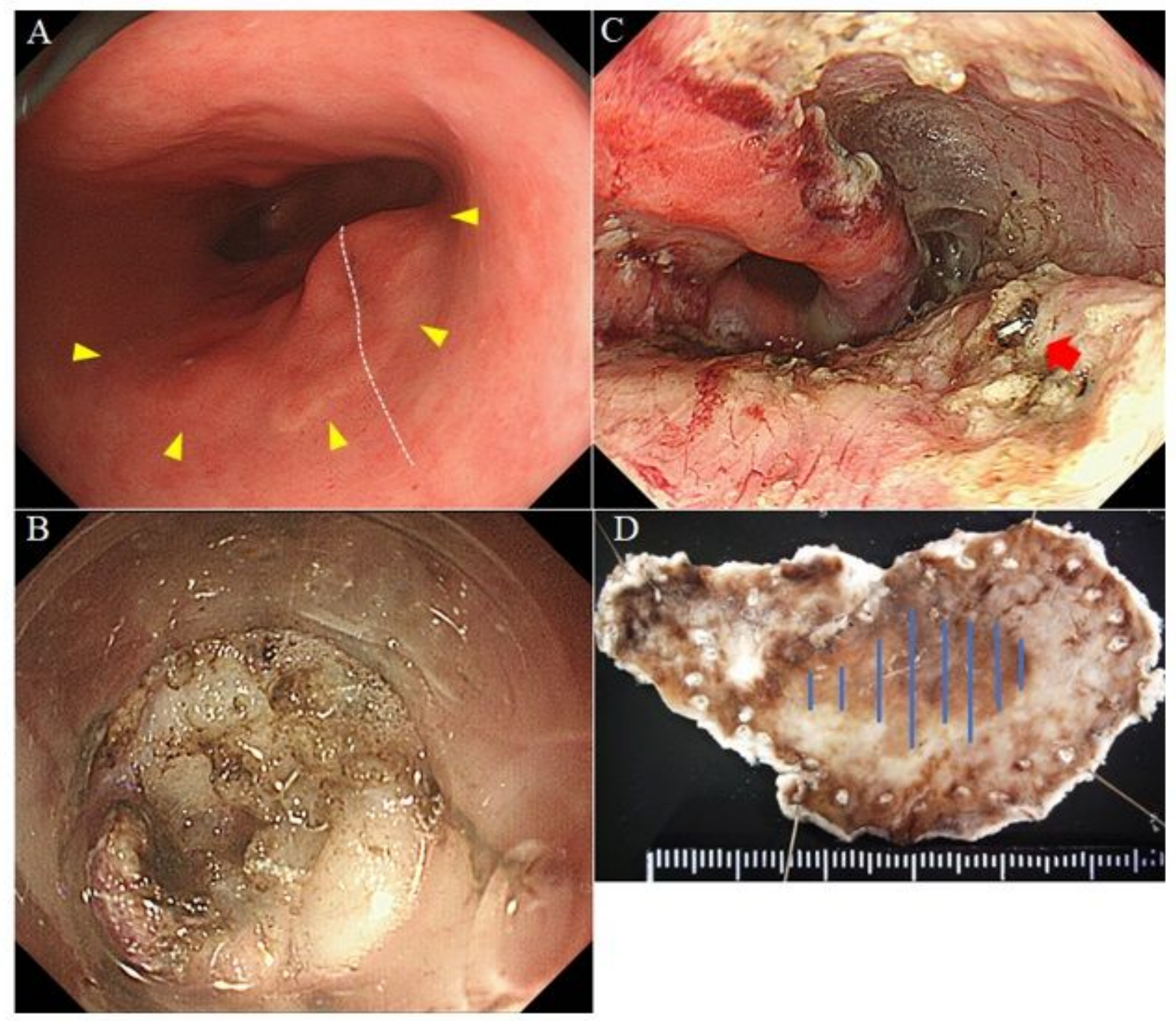

Figure 3

ESD for gastric tube carcinoma. (A) A tumor with a slightly depressed reddish lesion (0-IIc) is widespread and straddling the suture line (white dotted line). Yellow triangles indicate the tumor margin. (B) The submucosa requiring dissection has poor visibility and hard tissue because of severe fibrosis. (C) Endoscopic picture after ESD. Red arrow shows the remaining surgical staple. (D) Resected specimen. Blue lines indicate mucosal well-differentiated tubular adenocarcinoma. 


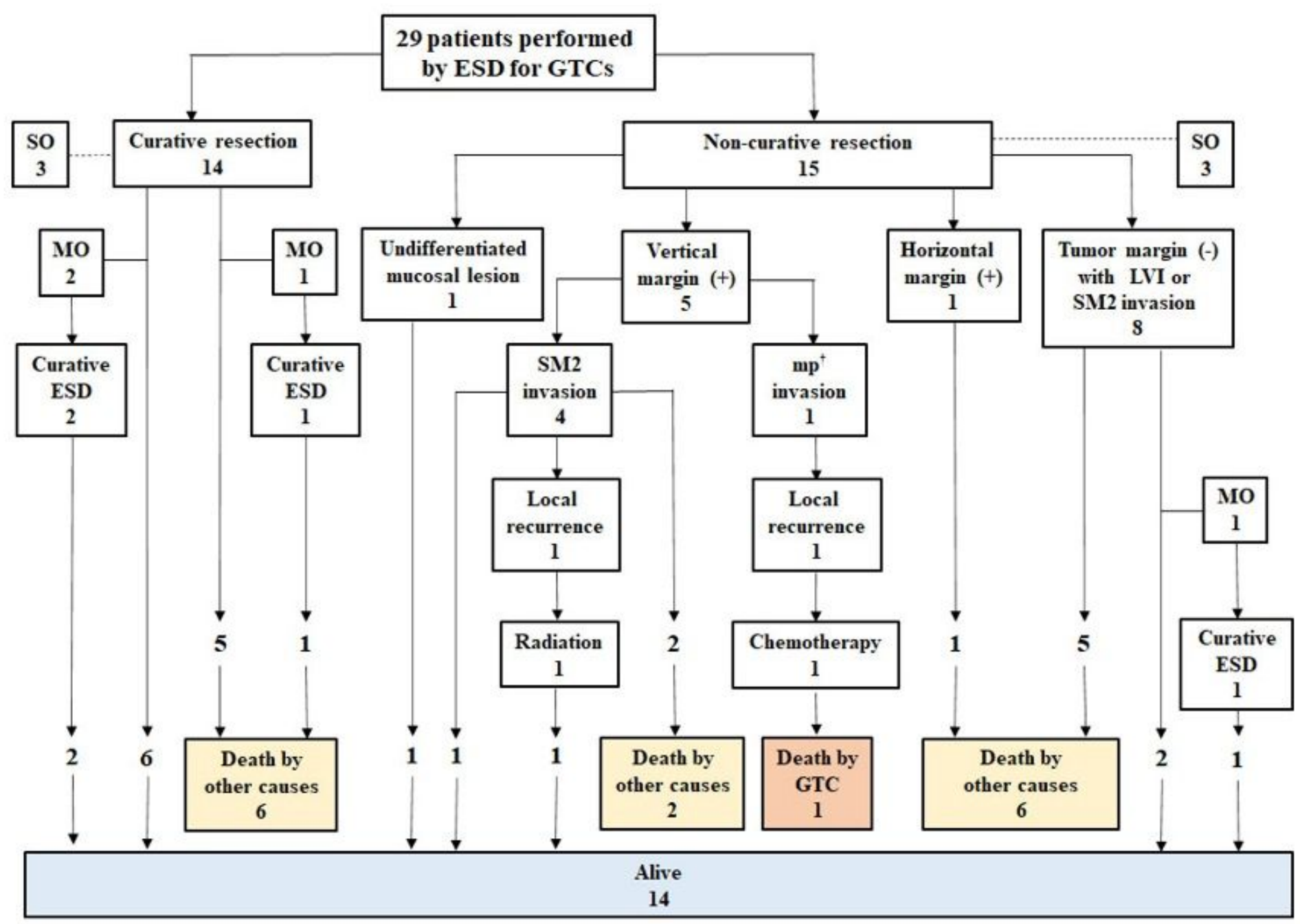

Figure 4

Clinical course after endoscopic submucosal dissection for gastric tube carcinoma. Among the 14 patients with curative resection, 8 patients were alive and 6 patients died of another disease. Fifteen patients with non-curative resection were followed-up without additional surgery. Among them, two patients with positive vertical margins had local recurrence and one died of the primary carcinoma. Eight patients died of another disease. MO: metachronous occurrence; SO: synchronous occurrence; LVI: lymphovascular invasion; SM2: $500 \mu \mathrm{m}$ or deeper invasion from the submucosa; MP: muscularis propria 

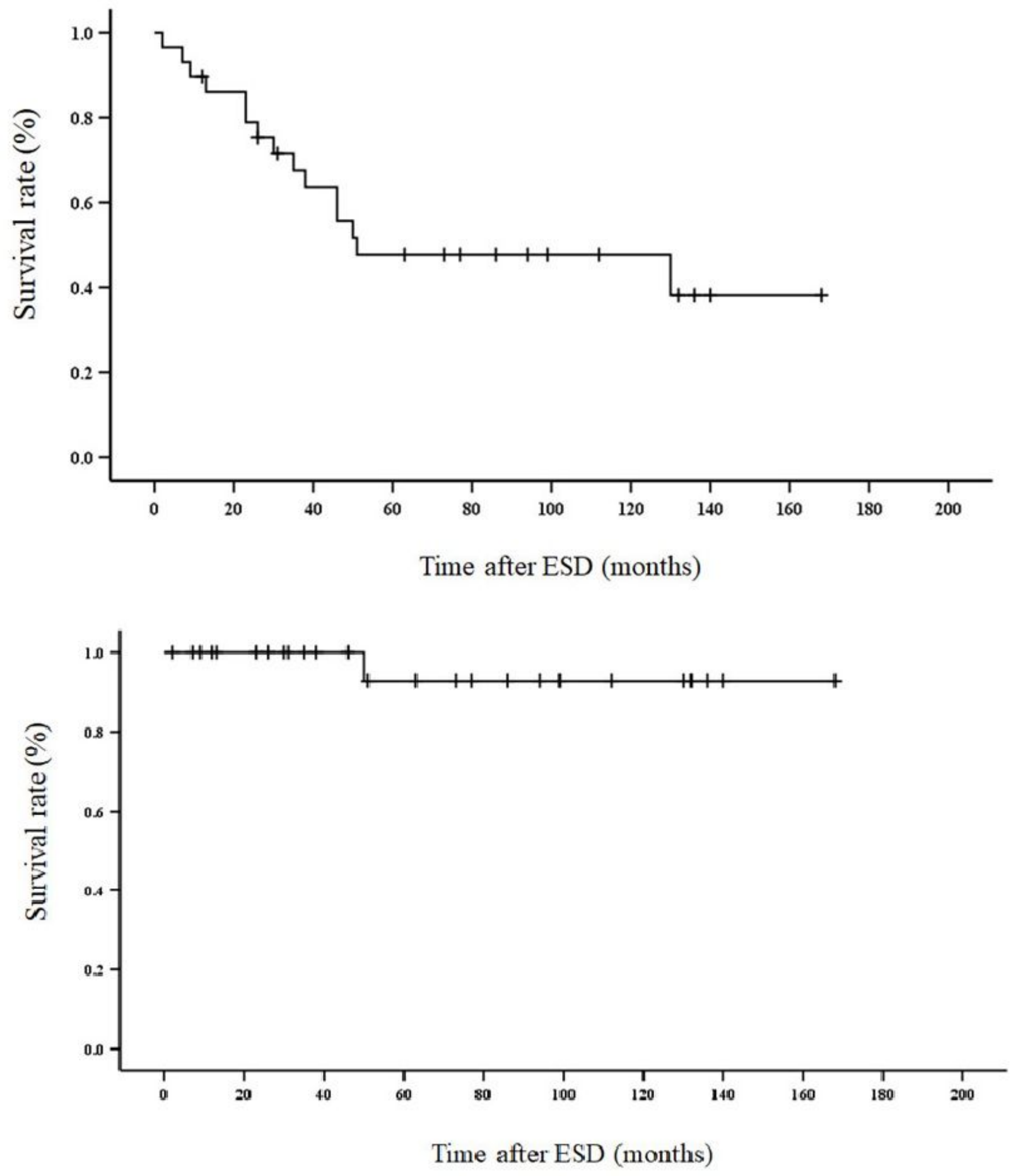

Figure 5

Long-term outcomes of the patients with gastric tube carcinoma treated with endoscopic submucosal dissection. (A) Overall survival. (B) Disease-specific survival. ESD: endoscopic submucosal dissection 